\title{
Correction to: a predictive model for women's assisted fecundity before starting the first IVF/ICSI treatment cycle
}

\author{
Juan J. Tarín ${ }^{1,2}$ (1) Eva Pascual ${ }^{1}$ (1) - Miguel A. García-Pérez ${ }^{2,3}$ (D) - Raúl Gómez ${ }^{2}$ (D) - Juan J. Hidalgo-Mora ${ }^{2,4}$ (1) \\ Antonio Cano ${ }^{2,4,5}$
}

Published online: 23 December 2019

(C) Springer Science+Business Media, LLC, part of Springer Nature 2019

\section{Correction to: Journal of Assisted Reproduction and Genetics.} https://doi.org/10.1007/s10815-019-01642-3

The original article unfortunately contained a mistake. In Table 2, the headers "Development set" and "Validation set" were not aligned to to their sub-headers.

The original version has been corrected.

The online version of the original article can be found at https://doi.org/ 10.1007/s10815-019-01642-3

\section{Juan J. Tarín}

tarinjj@uv.es

1 Department of Cellular Biology, Functional Biology and Physical

Anthropology, Faculty of Biological Sciences, University of

Valencia, Dr. Moliner 50, 46100 Burjassot, Valencia, Spain

2 Institute of Health Research INCLIVA, Valencia, Spain

3 Department of Genetics, Faculty of Biological Sciences, University of Valencia, Burjassot, Valencia, Spain

4 Service of Obstetrics an,d Gynecology, University Clinic Hospital, Valencia, Spain

5 Department of Pediatrics, Obstetrics and Gynecology, Faculty of Medicine, University of Valencia, Valencia, Spain 